Draft VERsion June 7, 2021

Preprint typeset using IATEX style emulateapj v. 03/07/07

\title{
THE FORMATION OF POLAR DISK GALAXIES
}

\author{
Chris B. Brook ${ }^{1}$, Fabio Governato ${ }^{1}$, Thomas Quinn ${ }^{1}$, James Wadsley ${ }^{2}$ \\ Alyson M. Brooks ${ }^{1}$, Beth Willman ${ }^{3}$, Adrienne Stilp ${ }^{1}$, Patrik Jonsson ${ }^{4}$ \\ Draft version June 7, 2021
}

\begin{abstract}
Polar Ring Galaxies, such as NGC4650A, are a class of galaxy which have two kinematically distinct components that are inclined by almost 90 degrees to each other. These striking galaxies challenge our understanding of how galaxies form; the origin of their distinct components has remained uncertain, and the subject of much debate. We use high-resolution cosmological simulations of galaxy formation to show that Polar Ring Galaxies are simply an extreme example of the angular moment misalignment that occurs during the hierarchical structure formation characteristic of Cold Dark Matter cosmology. In our model, Polar Ring Galaxies form through the continuous accretion of gas whose angular momentum is misaligned with the central galaxy.
\end{abstract}

Subject headings: cosmology:theory — galaxy:evolution — galaxy:formation

\section{INTRODUCTION}

Significant numbers of galaxies are known to have more than one kinematically distinct component, distinguishing them as "multi-spin" galaxies (Rubin 1994). In some cases, these distinct kinematic structures are essentially perpendicular to each other, and such galaxies are traditionally referred to as Polar Ring Galaxies (Whitmore et al. 1990). The formation of these exotic astronomical phenomena has been the subject of much debate (Casertano Sackett \& Briggs 1991), and their existence has presented a logical puzzle to the way we believe that galaxies may form. Interest in Polar Ring Galaxies is further heightened by their use in probing the nature of Dark Matter (Schweizer Whitmore \& Ruben 1983 Sackett el al. 1994; Iodice et al. 2006). Although it comprises $85 \%$ of the matter in the Universe, we know little about the nature of Dark Matter. Vital clues can be found by probing its role in forming these fascinating and quixotic galaxies which have two perpendicular components.

The most extensively observed Polar Ring Galaxies share many properties with disk galaxies, including exponential light profiles (Schweizer Whitmore \& Ruben 1983), large amounts (several times $10^{9} M_{\odot}$ ) of neutral Hydrogen (HI) (van Driel et al. 2000; Arnaboldi et al. 1995) in extended rather than narrow ring structures (van Gorkom Scechter \& Kristian 1987; van Driel et al. 1995; Arnaboldi et al. 1997; van Driel et al. 2000; Iodice et al. 2002; Gallagher et al. 2002) a ratio of HI mass to luminosity in the B band $(\mathrm{M}(\mathrm{HI}) / \mathrm{LB})$ typical of late-type spirals (Huchtmeier 1997; Arnaboldi et al. 1997; Sparke \& Cox 2000; Cox Sparke \& van Moorsel 2006), young stellar populations (Gallagher et al. 2002; Karataeva et al. 2004a; Cox Sparke \& van Moorsel 2006), ongo-

\footnotetext{
${ }^{1}$ Department of Astronomy, University of Washington, Box 351580, Seattle, WA 98195, USA

2 Department of Physics and Astronomy, McMaster University, Hamilton, ON, L88 4M1, Canada

${ }^{3}$ Harvard-Smithsonian Centre for Astrophysics, Cambridge, MA (Clay Fellow)

${ }^{4}$ Physics Department, University of California Santa Cruz, CA 95064, USA
}

ing and continuous rather than bursty star formation (Reshetnikov Faundez-Abans \& de Oliviera-Abans 2002; Karataeva et al. 2004b), disk galaxy colors and color gradients (Reshetnikov Hagen-Thorn \& Yakovleva 1994; Arnaboldi et al. 1995), flat rotation curves (Reshetnikov Faundez-Abans \& de Oliviera-Abans 2002; Swaters \& Rubin 2003), chemical abundances typical of disk galaxies (Buttiglione Arnaboldi \& Iodice 2006), and spiral arms (Arnaboldi et al. 1995; Iodice et al. 2004; Cox Sparke \& van Moorsel 2006). So compelling has been the evidence linking polar structures to disks in galaxies such as NGC4650A that a change of nomenclature is occurring, with increasing reference to Polar Disk Galaxies rather than Polar Ring Galaxies (Iodice et al. 2006). This change in nomenclature is strongly supported by our study; for the remainder of this paper we refer to these galaxies as Polar Disk Galaxies.

The two scenarios for the formation of Polar Disk Galaxies which have dominated the literature involve the interaction of two galaxies. One proposes that they form in a collisional merger between two galaxies (Bekki 1998). In the second scenario, the polar disk forms from gas which is stripped from a donor galaxy which passes by the central galaxy, without merging (Schweizer Whitmore \& Ruben 1983). Neither of the proposed galaxy interaction models, merger nor accretion from companions, has been shown to self-consistently explain the high gas mass within the polar structure, its extended nature, and the spatial coincidence of stars with this gas (Karataeva et al. 2004a), with no evidence of interaction induced star bursts. The merger model is also difficult to reconcile with the presence of an inner disk as observed, for example, in NGC4650A (Iodice et al. 2004) (disks are commonly believed to be destroyed by mergers). Further, both models predict that polar disk galaxies would reside preferentially in environments in which galaxy interactions are common, such as galaxy groups, yet such an environmental dependence is not observed (Brocca Bettoni \& Galletta 1997). This is emphasized by the fact that, before being reclassified as a polar ring galaxy, NGC6822 was considered a typical isolated dwarf irregular (Demers Battinelli \& Kunkel 2006). A third scenario of cold accretion claims that polar rings 
can be formed from the accretion of filamentary cold gas (Macciò Moore \& Stadel 2006). A study of a galaxy formed in cosmological galaxy formation simulations demonstrated that it is possible for gas accretion to be perpendicular to the central galaxy. Yet, as with models of accretion scenarios, the simulated galaxy does not share the detailed features of observed Polar Disk Galaxies, and the issue of the formation of these galaxies ultimately remained unresolved.

As part of a project undertaken by our group at the $N$ body Shop aimed at simulating a large sample of galaxies in a cosmological context, a simulated galaxy was found (serendipitously) having two concentric, almost perpendicular disks. This simulated galaxy, run within the "concordant" $\Lambda$-cold dark matter cosmology, shares the detailed features of observed Polar Disk Galaxies. When comparing these properties of our simulated Polar Disk Galaxies to those observed, it is important to mimic the methods employed by observers as closely as possible. The techniques used in this study to artificially "observe" our simulated galaxy are state of the art. Using the age and metallicity information of the star particles in the simulations, we determine how the light of our simulated galaxy would appear when observed specific telescopes. Using the age and metallicity information of the star particles in the simulations, we determine how the light of our simulated galaxy would appear when observed by the specific telescope used to observe the real galaxy to which the comparison is made, including the important effects of dust. We make particularly detailed comparisons with NGC4650A, considered the prototypical Polar Disk Galaxy (Whitmore et al. 1990).

\section{METHODS}

\subsection{Simulation code}

We have used the fully parallel, N-body, smoothed particle hydrodynamics (SPH) code GASOLINE (Wadsley Stadel \& Quinn 2004) to compute the evolution of the collision-less and dissipative elements respectively. Here we outline its essential features, while the interested reader is referred to the literature for full details (Governato et al. 2007). GASOLINE computes gravitational forces using a treecode that employs multi-pole expansions to approximate the gravitational acceleration on each particle (Barnes \& Hut 1986). Time integration is carried out using the leapfrog method, which is a second-order symplectic integrator. In cosmological simulations, it is necessary to implement periodic boundary conditions, achieved in GASOLINE by employing a generalized Ewald hernquist method to fourth order.

$\mathrm{SPH}$ is a technique which uses particles to integrate fluid elements representing gas (Gingold \& Monaghan 1977; Monaghan 1992). GASOLINE is fully Lagrangian, spatially and temporally adaptive and efficient for large numbers of simulation particles. The code includes radiative cooling and accounts for the effect of a uniform background radiation field on the ionization and excitation state of the gas. The implemented cosmic ultraviolet background, following the Harrdt-Madau model (Haardt \& Madau 1996), includes photoionizing and photoheating rates produced by QSOs, galaxies and POPIII stars starting at $\mathrm{z}=10$, consistent with the combination of the 3rd year WMAP results and the Gunn-Peterson effect in the spectra of distant quasars (Alvarez et al. 2006). We use a standard cooling function for a primordial mixture of atomic hydrogen. The internal energy of the gas is integrated using the asymmetric formulation that gives results very close to those of a formalism that conserves entropy, but conserves energy better. Dissipation in shocks is modeled using the quadratic term of the Monaghan artificial viscosity (Wadsley Stadel \& Quinn 2004), with a Balsara (Balsara 1995) correction term to reduce excessive artificial shear viscosity.

A simple but physically motivated recipe (Stinson et al. 2006) describes star formation and the effects of subsequent energy feedback from supernovae. Adopted supernova and star formation efficiency parameters were previously tested, and we use a Kroupa Initial Mass Function (Kroupa 2001). Metal enrichment from both supernova types Ia \& II are followed based on yields from the literature (Raiteri Villata \& Navarro 1996). The simulation described in this paper was run at the San Diego Supercomputing Facility using up to 512 CPUs, for a total of about 500k CPU hours.

\subsection{Cosmological Initial Conditions}

The initial conditions for the galaxy described in this paper were obtained using the so called volume renormalization technique to achieve higher resolution in a region of interest. It is a higher resolution realization of one the simulations already presented elsewhere (Brooks et al. 2007). The virial mass of the halo that was selected at $z=0$ to be re-simulated at higher resolution is $1.6 \times 10^{11} \mathrm{M}_{\odot}$ (the virial mass is measured within the virial radius $\mathrm{R}_{v i r}$, the radius enclosing an overdensity of 100 times the critical density $\left.\rho_{\text {crit }}\right)$. The halo was originally selected within a low resolution dark matter only simulation run in a concordance, flat, $\Lambda$-dominated cosmology: $\Omega_{0}=0.3, \lambda=0.7, h=70, \sigma_{8}=0.81$, where $\Omega_{0}$ is the matter density, $\Lambda$ the cosmological constant, $h$ is Hubbles constant, and $\sigma_{8}$ is the root mean square fluctuations in the matter power spectrum at the $8 \mathrm{Mpc}$ scale. The size of the simulated region, $28.5 \mathrm{Mpc}$, is large enough to provide realistic torques. The power spectra to model the initial linear density field were calculated using the CMBFAST code to generate transfer functions. Dark matter, star and gas particle masses in the highresolution regions are $9.4 \times 10^{4}, 3.3 \times 10^{3}$ and $1.6 \times 10^{4} \mathrm{M}_{\odot}$, respectively. The force resolution, i.e. the gravitational softening, is $0.15 \mathrm{kpc}$. In total there are $1.4 \times 10^{6} \mathrm{DM}$ particles within the virial radius. At $z=0$ there are a total of $3.5 \times 10^{6}$ baryonic particles within the central 20 kpc (each gas particle spawns up to three star particles and is then distributed between gas neighbors when its mass falls below $20 \%$ its original one). Star particles lose mass due to supernovae and stellar winds. This mass is redistributed to nearby gas particles. With our choices of particle number and softening, the smallest subhalos resolved have typical circular velocities of $10 \%$ of their host, ensuring that all subhalos able to retain even a small fraction of their baryons are resolved. The UV field makes ambient gas too hot to be bound to the lowest mass halos: most subhalos with mass below $5 \times 10^{9} \mathrm{M}_{\odot}$ are empty of stars. Particles in the high resolution region have gravitational spline softening evolved in a comoving manner from the starting redshift $(z=100)$ until $z=9$, and kept 


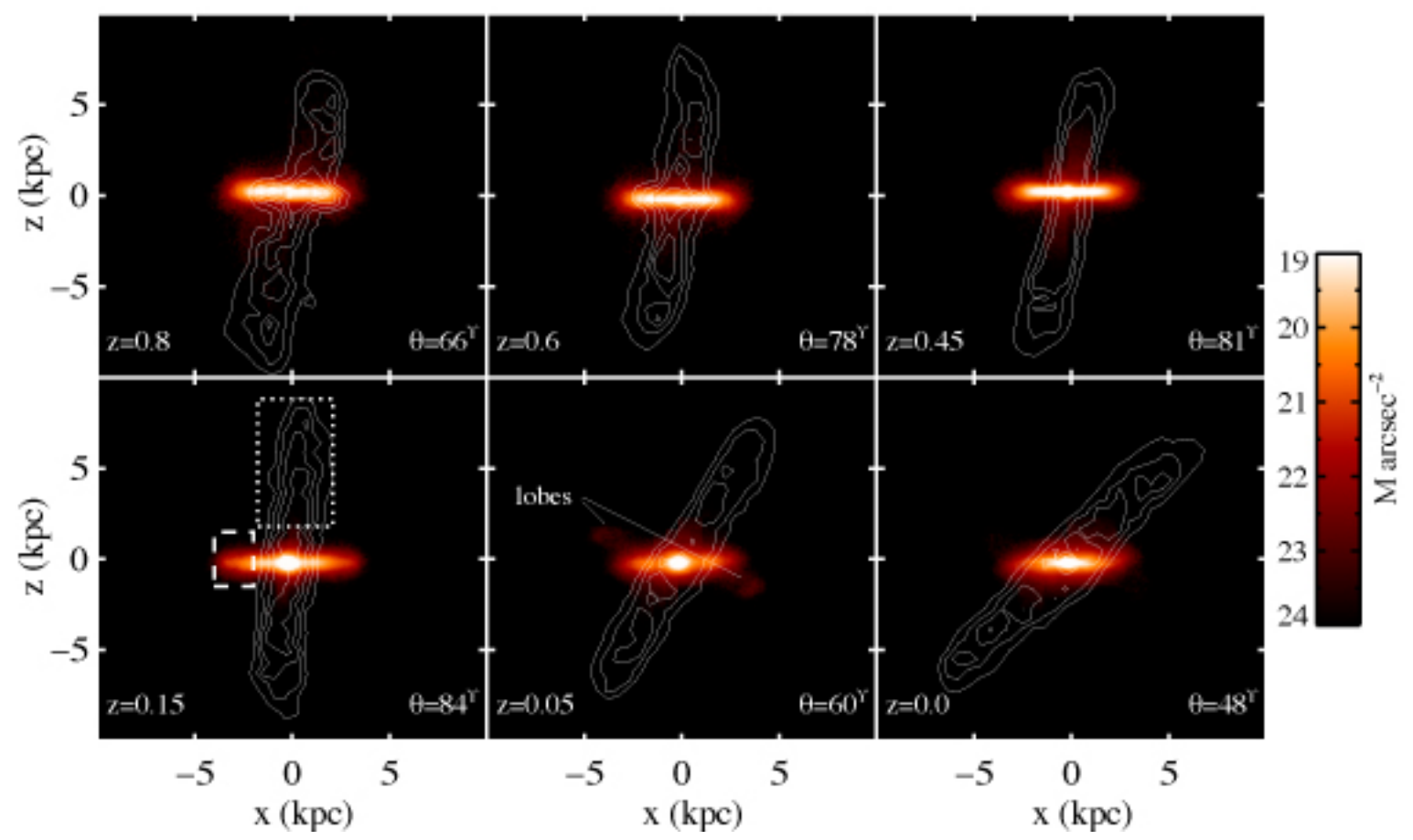

Fig. 1.- At six time-steps during the polar disk evolution, we make edge-on surface brightness maps of the inner disk stars, i.e. that form between redshift $z=1.6$ and $z=0.7$, in the SDSS i-band filter. The color bar indicates the surface brightness level in Magnitudes/arcsec ${ }^{2}$. We overplot a contour map of the cold gas $\left(<4 \times 10^{4}\right.$ Kelvin), where star formation takes place. At $z=0.8$, cold gas and hence ongoing star formation are present in the inner disk, but new cold gas accretion is highly inclined to this inner disk. The angle between this new forming polar disk and the inner disk is currently $66^{\circ}$, as indicated in the bottom right corner. Between $z=0.6$ and $z=0.15$, the cold gas remains at an inclination of between $77-84^{\circ}$ to the inner disk. The dashed box region at $z=0.15$ is selected for further analysis in Figure 2 as representative of the inner disk, whilst representative polar disk stars are within the dotted box. At $z=0.05$, a dynamically induced morphological feature becomes apparent in the inner disk, marked as lobes. By the present time, $z=0$, the polar and inner disk appear on their way toward aligning.

then fixed from $z=9$ to the present. The softening values are a compromise between reducing two body relaxation and ensuring spatial resolution of disk scale lengths and the central part of dark matter halos. The simulation was run with an integration parameter $\eta=0.195$ and a treecode opening angle $\theta$ of 0.525 for redshift $z>2$ and 0.725 afterwards (Moore et al. 1998, Power et al. 2003). The simulated galaxy analyzed in this study is the highest resolution of its kind in the published literature to date.

\subsection{Creation of Mock Images}

We used the open source software Sunrise (Jonsson 2006) to generate our artificial optical images in Fig. 1, 3, 4, $7 \&$ \&. Sunrise allows us to measure the dust reprocessed spectral energy distribution (SED) of every resolution element of our simulated galaxies from the far UV to the far IR with a full 3D treatment of radiative transfer. Filters mimicking those on major telescopes such as the Hubble Space Telescope (HST) are used to create mock observations. Sunrise uses Monte Carlo techniques to calculate radiation transfer through astronomical dust, including such effects in the determination of SEDs.

\section{EVOLUTION OF THE POLAR DISK}

Polar Disk Galaxies are only observed in the nearby Universe, due to inherent difficulties of observing these exotic galaxies, but they are presently in a variety of evolutionary stages. This allows us to link properties of observed Polar Disk Galaxies to different phases in the simulation, and unravel a detailed history of their evolution. In the particular merging history of the simulation in which a polar disk forms, an inner disk starts forming shortly after the last major merger at redshift $z \sim 2$. The mass ratio of the merging galaxies is very close to 1:1 but, due to its gas rich nature, the galaxy rapidly forms a new disk whose angular momentum is largely determined by the merger orbital parameters. At later times gas continues to be accreted to the galaxy but in a plane that is almost perpendicular to the inner disk.

We first analyze a snapshot of the galaxy at redshift $z \sim 0.8$, shortly after the polar disk begins forming. At this time, the central galaxy is still forming stars in a disk, while the bulk of new star formation is in the highly inclined polar disk $\left(\sim 66^{\circ}\right.$ to the inner disk, Fig. 1$)$, with star formation continuing in both disks until $z=0.6$ (see Fig. 2). Two prominent spiral arms are evident in the polar structure. At this stage, the galaxy shares many features of NGC660, a local polar ring galaxy in which the central galaxy is still forming stars. The polar structure in both NGC660 and the simulation is a disk with an exponential light profile rather than a ring, and has a young stellar population with continuous star formation (van Driel et al. 2000). By $z=0.5$, the inner disk has exhausted its gas, whilst gas continues to fall onto the polar disk. From this point in time to the present, the galaxys star formation occurs almost exclusively in the polar disk (Fig. 2). The polar disk continues to accrete material, at an inclination which remains stable between $78-84^{\circ}$ until $z=0.15$. Almost all of the 


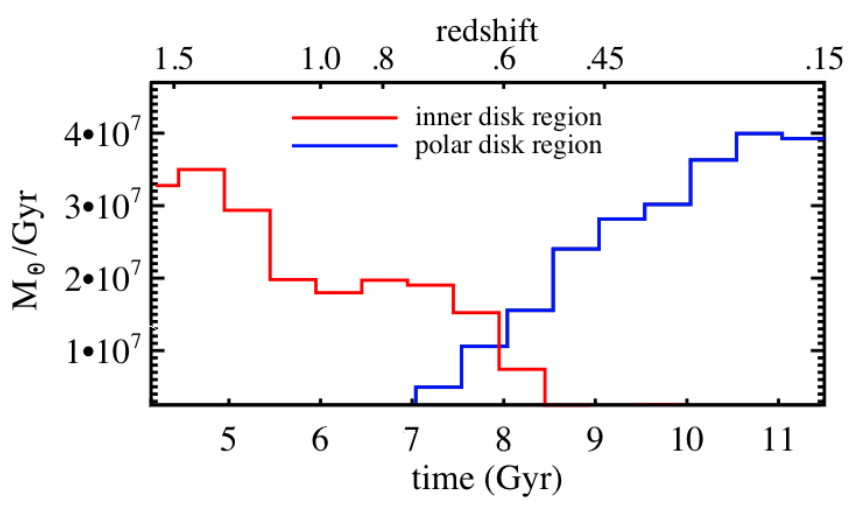

FIG. 2.- Stars from a region of the inner disk at $z=0.15$ (dashed box, Fig. 1) and polar disk (dotted box, Fig. 1) are selected. We plot the star formation history within the two regions, since the time when the inner disk begins to form. Stars form reasonably steadily within the inner disk until $z \sim 0.7$, and cease by $z \sim 0.5$. This reflects the lack of cold gas in the inner disk region at later times, shown in Fig. 1. The polar disk stars began forming at $z \sim 0.7$, with star formation relatively constant for 1.5 Gyrs between $z \sim 0.5$ and $z \sim 0.3$, and then constant at a slightly higher rate for a further 1.5 Gyrs between $z \sim 0.3$ and $z \sim 0.15$.

neutral hydrogen, as seen in Fig. 1, is perpendicular to the inner disk during this time. The likeness to the HI maps of UGC9796 (Cox Sparke \& van Moorsel 2006), and UGC7575 (Sparke \& Cox 2000), when also overplotted on optical images which highlight their inner disk, is striking (see also section 4.3).

The polar disk in our simulation is stable for at least the 3 Gyrs between $z=0.6$ and $z=0.15$. This is well supported by observations of stellar populations in several polar disks, which indicate that such structures are long lived. For example, the stellar population of the polar disk in NGC4650A has most likely been continuously forming for stars 3 Grys (Gallagher et al. 2002 Mould et al. 1982), and the star formation observed in the polar disk of UGC9796 indicates that it is 3-5 Gyrs old (Cox Sparke \& van Moorsel 2006). Fig. 3 shows the stunning correspondence between the simulated Polar Disk Galaxy at $z=0.15$ and NGC4650A. At this time, the simulated Polar Disk Galaxy and NGC4650A both have extended polar disk structures with young stellar populations, ongoing star formation, blue colors, spiral arms, $M(H I) / L_{B}$ ratios that are typical of low surface brightness galaxies (Iodice et al. 2002), and almost flat rotation curves. Both have rotationally supported S0 like inner galaxies, which have had little or no star formation over a period of 3 Gyrs, and exponential light profiles (Iodice et al. 2004). Details of these properties of the inner and polar disks of the simulation are presented in section 4.

During the final 1.5 Gyrs to the present, our simulated polar disk becomes less stable, and in particular the inner regions of the polar disk begin to dynamically interact with stars in the central galaxy. This creates a complex dynamical system with the polar and inner structures becoming less distinct. The inner regions of the Polar Disk Galaxy ESO-603-G21 show similarly complex and overlapping dynamical structures (Reshetnikov Faundez-Abans \& de Oliviera-Abans 2002). At $z \sim 0.05$, the inner disk of our simulated galaxy forms a morphological feature remarkably similar to
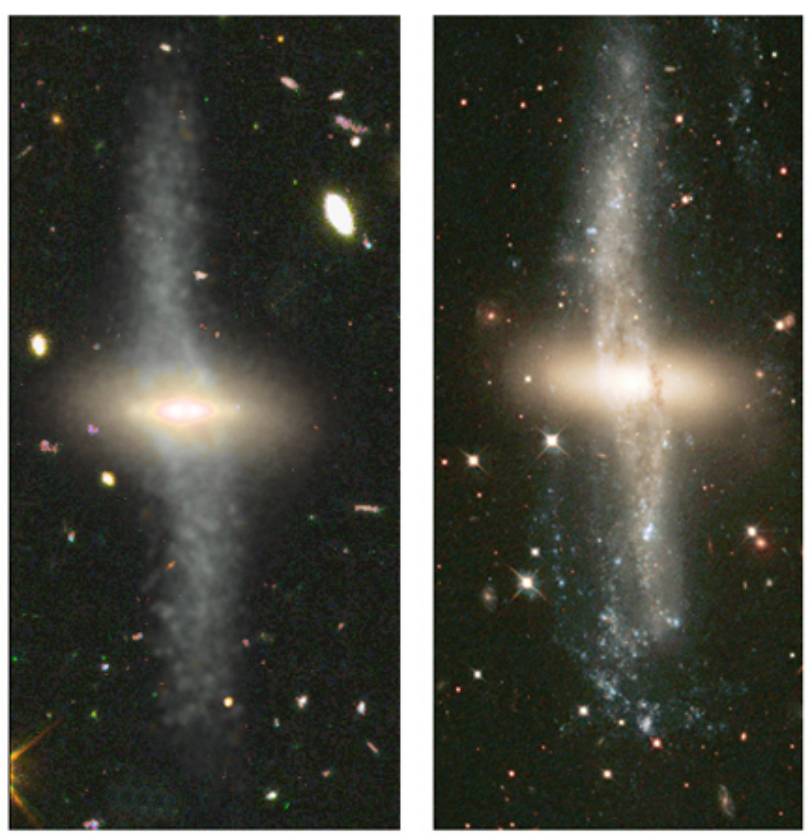

FIG. 3.- The simulated polar disk (left) is imaged by assigning three mock HST bands, at 450, 606, and $814 \mathrm{~nm}$, the colors blue, green and red, respectively. The image is superimposed onto an HST background. NGC4650A, considered the prototypical polar disk galaxy, is imaged (right) using the same color-band assignment (Gallagher et al. 2002). The red color of the inner disks reflects the old age of their stars, which emit longer wavelength light. The younger stars of the polar disks are more prominent in the shorter wavelength bands, hence their blue hue.

those described as "lobes" in the central galaxy of ESO-603-G21 (Fig. 1). These are old stars that have been dynamically stirred from the inner disk by the interaction of the two disks during this stage of the galaxys evolution. Interestingly, ESO-603-G21 also has a polar disk that is prominent in the K-band (Reshetnikov Faundez-Abans \& de Oliviera-Abans 2002), indicating that, like the polar disk component of our simulation at this time, it also is several Gyrs old.

\section{PROPERTIES OF THE INNER AND POLAR DISKS}

We refer to various properties of the two orthogonal components of our simulated polar disk galaxy in the previous section, in which we follow the galaxy's evolution. We show plots which verify these properties in this section, presented in a manner which closely mimics observations, facilitating direct comparisons with observed polar ring galaxies. In several plots, we provide distances in kiloparsecs as well as arcseconds. Arcsecond scales are derived by assuming that the simulated galaxy has the same heliocentric systemic velocity as observed for NGC4650A (Arnaboldi et al. 1997), and using $70 \mathrm{~km} / \mathrm{s} / \mathrm{Mpc}$ for the Hubble constant, which implies that $1^{\prime \prime}=201 \mathrm{pc}$. Table 1 provides a summary of the properties of the simulated galaxy, its inner (S0 type) disk and the polar disk at $z=0.15$, when the simulation most resembles NGC4250A

\subsection{Light Profiles}

The radial surface brightness profiles of both components of our simulated polar disk galaxy attest to their disk galaxy nature. We plot these profiles, in Fig. 5 , in 


\begin{tabular}{ccccccccc}
\hline \hline & $\mathrm{I}_{A B}^{a}$ & B-I $^{b}$ & $\begin{array}{c}\operatorname{Vrot}^{c} \\
\mathrm{~km} / \mathrm{s}\end{array}$ & $\mathrm{V} / \sigma_{V^{d}}$ & $\begin{array}{c}\mathrm{h}_{l}{ }^{e} \\
\mathrm{kpc}\end{array}$ & $\begin{array}{c}\text { Stellar Mass }^{f} \\
\mathrm{M}_{\odot}\end{array}$ & $\begin{array}{c}\mathrm{HI} \text { Mass } \\
\mathrm{M}_{\odot}\end{array}$ & $\begin{array}{c}\mathrm{M}(\mathrm{HI}) / \mathrm{L}_{B}^{g} \\
\mathrm{M}_{\odot} / \mathrm{L}_{\odot}\end{array}$ \\
\hline Galaxy & -20.3 & 1.7 & - & - & - & $1.0 \times 10^{10}$ & $2.1 \times 10^{9}$ & 0.80 \\
Inner & -19.8 & 1.6 & 105 & 4.4 & 0.95 & $3.5 \times 10^{9}$ & - & - \\
Polar & -19.2 & 1.3 & 115 & 1.4 & 3.4 & $1.6 \times 10^{9}$ & $2.1 \times 10^{9}$ & 1.4 \\
\hline
\end{tabular}

TABLE 1

Summary of the properties of the simulated galaxy,its central S0 galaxy and the polar disk at $z=0.15$, when the simulation most resembles NGC4250A. (a) inner and polar absolute magnitudes are derived by calculating contributions to flux of stars born before and after $z=0.8$, after which star formation is mainly in the polar disk. (b) Inner and polar disk colors are for the regions indicated at $z=0.15$ in Fig. 1 (c) Line of sight velocity at 2.2 scalelengths (see Fig. 6), (d) at 2.2 scalelengths, (e) see Fig. 5 (f) a significant amount of galaxy stellar mass is in an old, faint bulge/spheroid, (g) for the region of the polar disk indicated at $z=0.15$ in Fig. 5

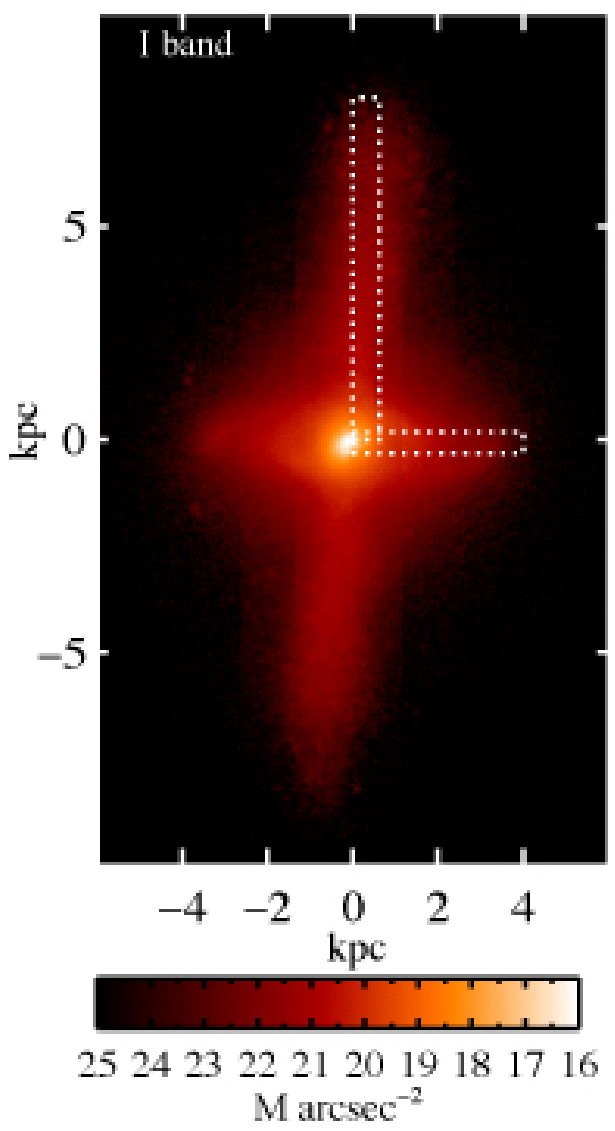

Fig. 4.- The two perpendicular regions over which surface brightness profiles are taken in Fig. 5 are indicated by the rectangles in this I-band surface brightness map. These regions are also used for deriving line of sight rotation curves in Fig. 6

two perpendicular regions as indicated in the left panel, at $z=0.15$. Both components have exponential light profiles outside the central bulge, with scalelengths of the inner and polar disks of approximately 0.95 and 3.4 kpc, respectively. In order to facilitate easy comparison with observations (Iodice et al. 2002), we assume that the galaxy is at the same distance as NGC4650A. The radius in arcseconds indicated in the upper axis of the plot.

\subsection{Rotation Curves}

We derive rotation curves of the inner and polar disk galaxies by using line of sight velocities of the stars and cold gas $(\mathrm{T}<40,000 \mathrm{~K})$ respectively, in the regions indicated in Fig. 5. Both the inner disk and polar disk rotation curves become reasonably flat. The rotation velocity at 2.2 scalelengths is very similar for both com-

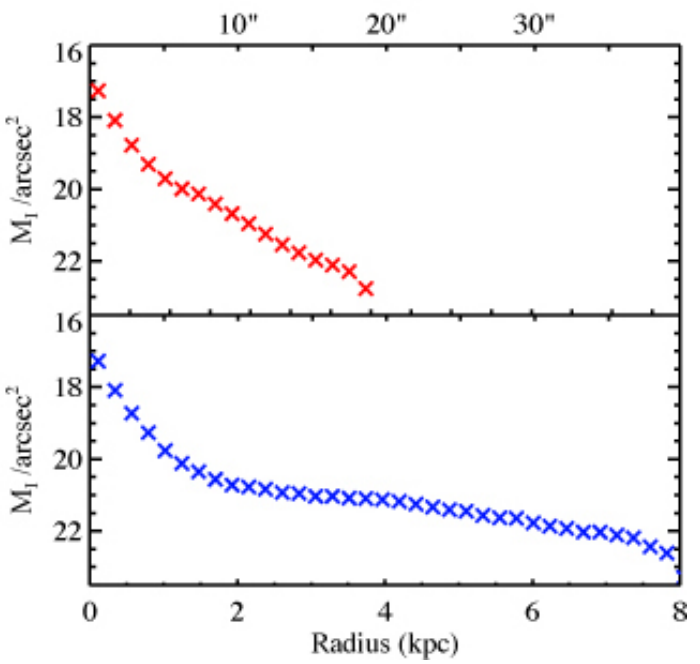

FIG. 5.- Radial surface brightness profiles in the I-band are plotted for the central galaxy (red crosses, upper panel) and polar disk (blue, lower panel). The two perpendicular regions over which the profiles are taken are indicated by the rectangles in Fig. 4
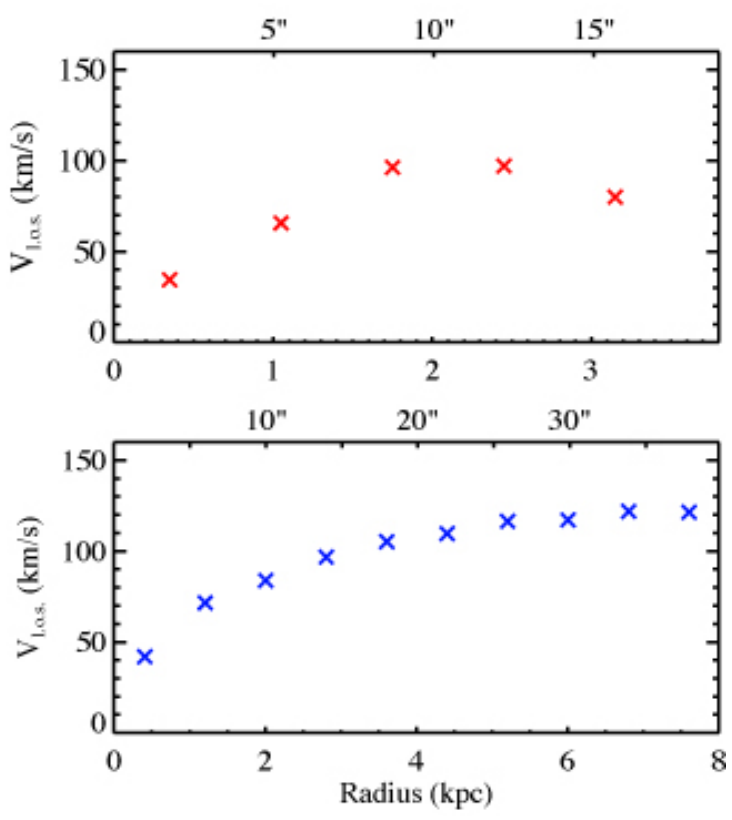

FIG. 6. - Line of sight velocity, versus radius, in the two regions indicated in Fig. 5. Radial distances are provided in both kpcs and arcseconds. The inner disk velocities (red crosses, upper panel) are derived using stars, while the polar disk velocities (blue crosses, lower panel) were derived using cold gas $(\mathrm{T}<40,000 \mathrm{~K})$. 


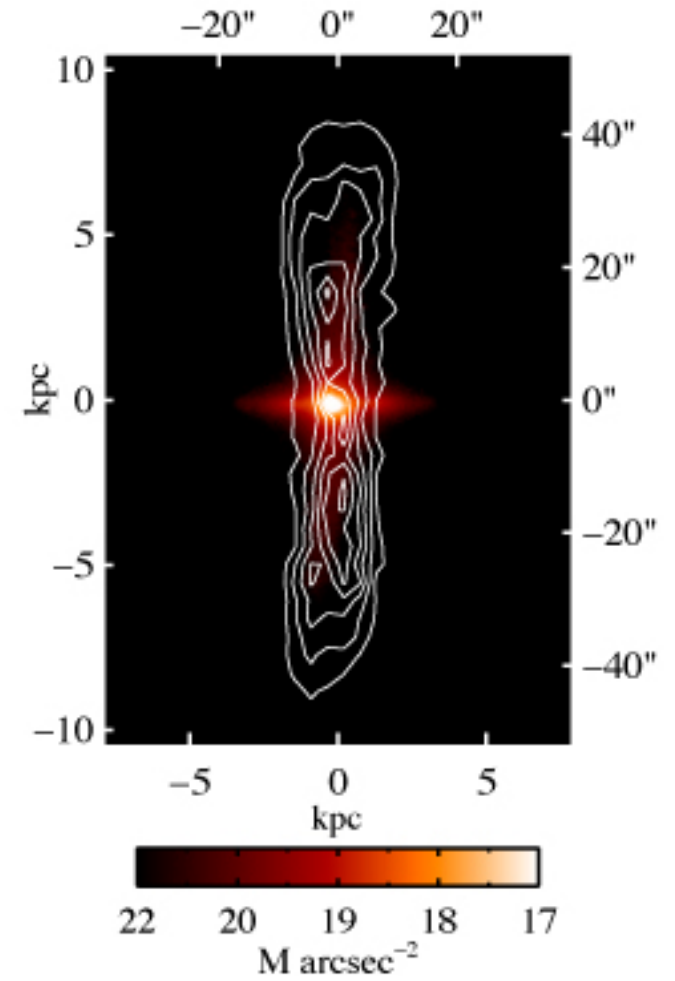

FIG. 7.- Surface brightness map of the simulation in the SDSS r-band with the HI density map overplotted, shown at $z=0.15$.

ponents, $\sim 105 \mathrm{~km} / \mathrm{s}$ and $\sim 115 \mathrm{~km} / \mathrm{s}$ for the inner and polar disks respectively. Radial distances are provided in $\mathrm{kpc}$ as well as arcseconds to facilitate comparison with observations (Swaters \& Rubin 2003, Iodice et al. 2006) of NGC4650A. Both components are rotationally supported; $V / \sigma_{V}$ at 2.2 scalelengths are 1.3 and 4.4 for the inner and polar disks respectively.

\subsection{HI warps and position-velocity maps}

The neutral Hydrogen (HI) maps of the simulations are plotted in Figs. 7 \& 8 . Fig. 7 is an SDSS r-band surface brightness image, which highlights the old, central galaxy stars, with contour lines of HI gas overlaid. Almost all the $\mathrm{HI}$ is in the polar disk, virtually perpendicular to the central galaxy. The simulation is shown here at $\mathrm{z}=0.15$, and presents greater detail than seen in Fig. 1. The morphological features of the HI maps are very similar to those observed in polar disk galaxies such as UGC9796 (Cox Sparke \& van Moorsel 2006) and UGC7575 (Sparke \& Cox 2000). An integral shaped warp is apparent in these HI maps of the simulated and observed polar disk galaxies. Such warps are evident at various times in the simulation.

The HI position-velocity map for simulation at $z=15$ is shown in Fig. 8, computed by projecting the data onto the major axis of the polar disk. Our simulated galaxy shares many qualitative features as the position-velocity map of NGC 4650A (Sparke \& Cox 2000), in particular the characteristics of a disk rather than ring: modeling an infinitely narrow ring would produce a linear positionvelocity diagram.

\subsection{Spiral Arms}

The polar disk of the simulated polar disk galaxy has its most prominent grand design spiral arms at early

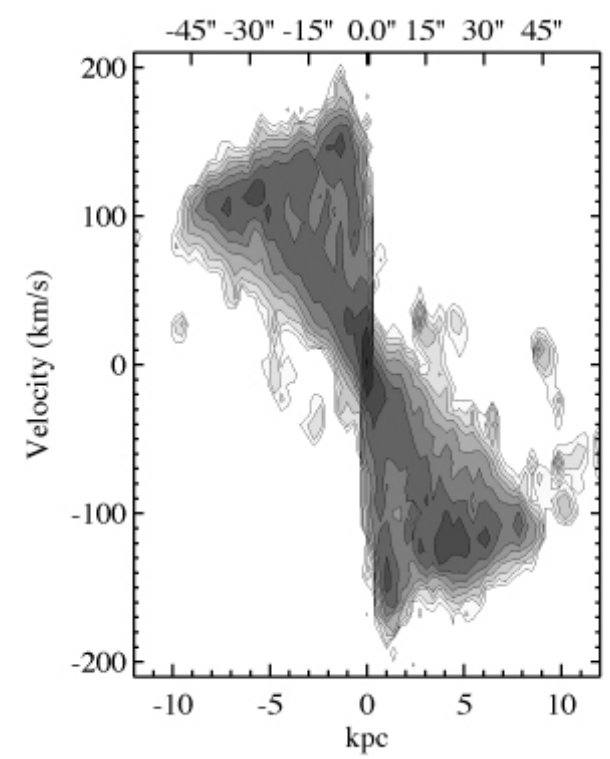

FIG. 8.- HI position-velocity map of the simulation at the same epoch.

times in its evolution. We highlight the two spiral arms as they exist at $z=0.6$ in the simulation, in Fig. 9. The surface brightness in the V-band image (panel A) is sensitive to recent star formation, and shows the polar disk at an angle of $40^{\circ}$ to edge on, and the central galaxy's inner disk almost edge on. The longer wavelength light imaged in the I-band (panel B) is more sensitive to older stellar populations. By subtracting the I-band image from the V-band, the difference, V-I image (panel C) highlights recent star formation, which occurs largely in the spiral arms. It is interesting that NGC4650A also has two grand design spiral arms (Arnaboldi et al. 1997).

\section{DISCUSSION}

The misalignments of angular momentum vectors between early and later accreted material is expected to be common in Cold Dark Matter structure formation, but in general the misalignment is small (Quinn \& Binney 1992). In all our disk galaxy simulations, we see smaller misalignment of the inner disk, and later forming outer disk, at some time during its formation, resulting in a transient "integral sign" warp in the galaxy. Such warps are seen in over half of observed disk galaxies (Bosma 1991), indicating that these processes of misalignment witnessed in our simulations are real. Polar disks are the rare cases of such processes where the inclination is extreme. Such polar inclinations are seen to be stable, as is the case in our simulation

It is telling that Polar Disk Galaxy ESO-325-G58, which is observed face on, was classified as a barred spiral before it was shown that the "bar" was in fact an inner disk perpendicular to the line of sight (Iodice et al. 2004). Similarly, NGC6822 is a local galaxy that was long classified as a barred irregular galaxy, but has recently been shown to be a polar ring galaxy (Demers Battinelli \& Kunkel 2006). An interesting observational challenge suggested by our study is to determine the number of face on Polar Disk Galaxies that have been classified as barred galaxies.

This scenario also has important implications for studying the nature of dark matter; Polar Disk 

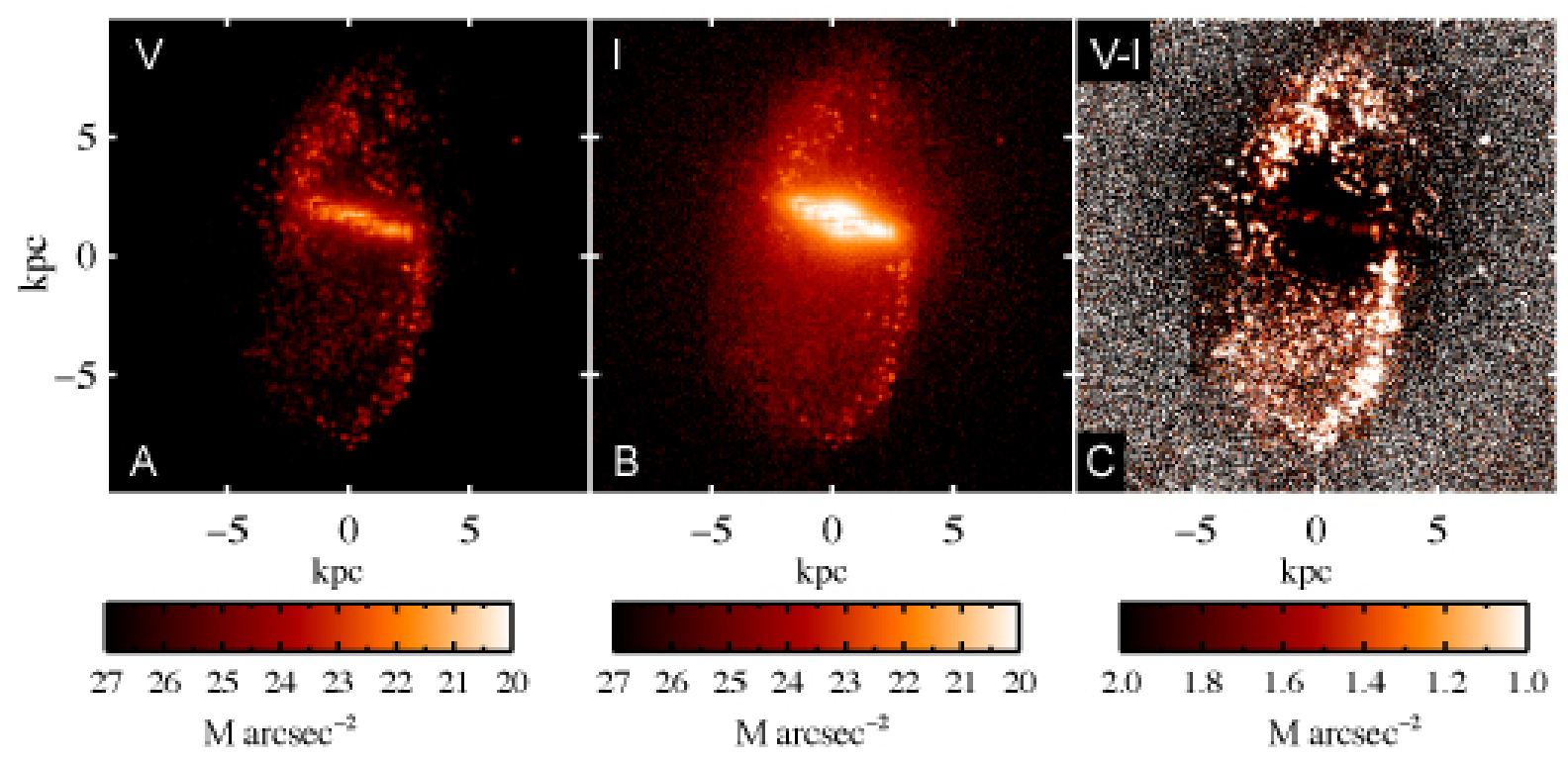

Fig. 9.- The V-band image (panel a) is sensitive to recent star formation, and shows the central galaxy's inner disk almost edge on. Two prominent spiral arms are evident in the polar structure. The longer wavelength light imaged in the I-band (panel b) is more sensitive to older stellar populations. The V-I image (panel c) is derived by subtracting I band image from the V-band, and highlights recent star formation.

Galaxies are used as a probe of the shape of dark matter halos (Casertano Sackett \& Briggs 1991; Schweizer Whitmore \& Ruben 1983; Sackett el al. 1994; Iodice et al. 2006). Our study implies that we now have the important added ability to determine how the shape of the dark halo is oriented with respect to the direction of cosmological gas flow, and how these relate to the surrounding distribution of matter in which a galaxy is embedded.

The processes of galaxy merging and accretion are both features of a Cold Dark Matter dominated universe and both are capable, indeed necessary, in producing observed properties of galaxies. The galaxies we have referred to in this study have been characterized by polar structures with disk galaxy characteristics, and we have shown that such galaxies form from cosmological gas infall. In other galaxies, such as AM 2020-504 (Whitmore et al. 1990, Arnaboldi et al. 1993), the polar structure is better described as a ring, with gas and stars in a narrow annulus. Deciding which galaxies are classified as polar rings and which as polar disks is an observational problem, which is best accomplished by comparing the polar structure to the outer regions of late type galaxies. Do Polar Ring and Polar Disk Galaxies form in the same manner? There are two ways in which narrow polar structures may form within our model for Polar Disk Galaxies. The passage of a small, satellite galaxy through a galactic disk is capable of transforming the disk into a ring. The spectacular "Cartwheel" galaxy is a prototype for Ring Galaxies (Zwicky 1941). This mechanism is capable of forming a Polar Ring Galaxy from a Polar Disk Galaxy. It is also possible that gas infall along a filament may have a narrow angular momentum distri- bution, and naturally settle into a ring. But our study is not conclusive on this issue, and the classic merging and accretion models remain viable explanations for the formation of narrow polar ring structures. For now, it may be necessary to treat Polar Rings and Polar Disks as two distinct galaxy types.

Most of the comprehensively studied polar structures are disks, and the evidence linking the polar disk structures to normal disks is compelling. The irresistible conclusion of our simulation is that the formation processes of Polar Disk Galaxies are the same as for the outer disk regions of normal disk galaxies, except the polar disk forms in a different plane to the central galaxy. Our scenario naturally explains the properties of several extensively studied Polar Disk Galaxies in terms of the different stages of their evolution. The notion of "rebuilding a disk" is an essential ingredient in galaxy formation models, and has been previously demonstrated in simulations (Steinmetz \& Navarro 2002). Our study implies that Polar Disk Galaxies are a spectacular demonstration of the rebuilding of disks through accretion of gas from the "cosmic web" predicted by hierarchical models of galaxy formation

We thank Enrica Iodice for helpful discussions, and Brad Gibson and Julianne Dalcanton for feedback on a draft. C.B., F.G., T.Q. \& A.B. are supported by an NSF ITR grant, PHY-0205413. F.G. was supported by NSF grant AST-0607819 and by a Spitzer Theory Grant. The simulation was run at the San Diego Supercomputing Facility.

\section{REFERENCES}

Alvarez, M.A., Shapiro, P., Kyungjin A. \& Iliev, I.T. 2006 Astrophys. J. 644 L101-L104
Arnaboldi, M., Capaccioli, M., Barbaro, G., Buson, L., Longo, G. 1993, A\&A, 268, 103 
Arnoboldi, M., Freeman, K., C., Sackett, P. D., Sparke, L., Capaccioli, M. 1995 Planet. Space Sci., 43, 1377

Arnoboldi, M., Oosterloo, T., Combes, F., Freeman, K., C., Koribalski, B., 1997 AJ, 113, 585

Balsara, D.S. J.Comput. Phys. 121 357-372 (1995)

Barnes, J., \& Hut, P. (1986) Nature 324, 446-449

Bekki, K. 1998 ApJ, 499, 635

Bosma, A. 1991 in Warped Disks and Inclined Rings around Galaxies, ed, S. Casertano, P. D. Sackett, \& H. F. Briggs 1991 (Cambridge: Cambridge Univ. Press), 181

Brocca, C., Bettoni, D., Galletta, G. 1997 A\&A, 326, 907-914

Brooks, A. et al. 2007 ApJ, 655, 17

Buttiglione, S., Arnaboldi, M., \& Iodice. E., 2006 Mem. A.S.It.Suppl. Vol. 9, 317

Casertano, S., Sackett, P. D., \& Briggs, F. H. 1991 Warped Disks and Inclined Rings around Galaxies, (Cambridge University Press: Cambridge)

Cox, A. L., Sparke, L. S., van Moorsel, G. 2006 AJ, 131, 828

Demers, S., Battinelli, P., \& Kunkel, W. E. 2006, ApJ 636, L85-88

Governato, F. et al. 2007 MNRAS, 374, 1479

Gallagher et al. 2002, ApJ, 568, 199

Gingold, R. A. \& Monaghan, J. J. 1977 Mon. Not. R. Astron. Soc. 181, 375-389

Haardt , F. \& Madau. P. 1996 Astrophys. J. 461, 20-37

Hernquist, L., Bouchet, F. \& Suto, Y. 1991 Astrophys. J. Supp 75, 231-240

Huchtmeier, W. K. 1997, A\&A, 319, 491-404

Iodice, E., Arnaboldi, M., Saglia, R. P., Sparke, L. S. Gerhard, L.S., Gallagher, J. S. Combes, F., Bournard F., Capaccioli, R. M., Freeman, K. C. 2006 ApJ, 643, 200

Iodice, E., Arnoboldi, M., Sparke, L. S., Gallagher J. S., Freeman, K. C. 2002 A\&A, 391, 103

Iodice, E., Arnoboldi, M., Sparke, L. S., Buta, R. M., Freeman, K. C. \& Capaccioli, A\&A, 418, 41

Jonsson, P. 2006 MNRAS, 372, 2

Karataeva, N. A. et al. 2004, AJ, 421, 833

Karataeva, N. A. et al. 2004, AJ, 127, 789
Kroupa, P., MNRAS, 322, 231, (2001)

Macciò, A. V., Moore, B. \& Stadel, J. 2006 ApJ, 636, 25

Monaghan, J.J. 1992 Annual. Rev. Astron, Astrophys. 30, 543-574

Moore, B., Governato, F., Quinn, T., Stadel, J., Lake, G. 1998 ApJ, 499,5

Mould, J., Balick, B., Bothun, G. \& Aaronson, M. 1982 ApJL 260, L37

Power, C. et al. 2003, MNRAS, 338, 14

Quinn, T. \& Binney, J. 1992 MNRAS, 255, 729

Raiteri, C. M., Villata, M. \& Navarro, J. F., A\&A, 315, 105 (1996)

Reshetnikov, V. P., Hagen-Thorn, V. A., \& Yakovleva, V. A. 1994 A\&A, 290, 693

Reshetnikov, V. P., Faundez-Abans, M. \& de Oliviera-Abans, M. 2002 A\&A 383, 390

Rubin, V. C. 1994, AJ, 108, 456

Sackett, P. D. Rix, H., Jarvis, B. J., Freeman, K. C. 1994, ApJ, 436, 629

Schweizer, F., Whitmore, B. C. \& Ruben, V. C. 1983 AJ, 88, 909

Sparke, L. S., \& Cox, A., L. 2000 in Dynamics of Galaxies: from early Universe to the Present ASP Conference Series, Vol. 197, eds. F. Combes, G. A. Mamon, and V. Charmandaris

Steinmetz, M. \& Navarro, J. F. 2002 NewA, 7, 155S

Stinson, G. Seth, A. Katz, N. Wadsley, J. Governato, F. Quinn, T. 2006 MNRAS, 373, 1074S

Swaters, R., A., \& Rubin, V., C. 2003 ApJ, 587, 23

va n Gorkom, J. H., Scechter, P. L., \& Kristian, J. 1987 ApJ, 314, 457

van Driel, W. et al. 1995 AJ, 109, 942

van Driel, W., Arnaboldi, M, Combes, F., Sparke, L. S. 2000 A\&AS, 141,385

Wadsley, J., Stadel, J., \& Quinn, T. 2004 New Astr. 9, 137-158

Whitmore, B. et al. 1990, AJ, 100, 1489

Zwicky, F. Theodore von Karman Anniversary Volume, Contribution to Applied Mechanics and Related Subjects (Pasadena: Caltech), 137, (1941) 